\title{
Quantifying the magnitude of pharyngeal obstruction during sleep using airflow shape
}

\author{
Dwayne L. Mann ${ }^{1,2}$, Philip I. Terrill ${ }^{1,2}$, Ali Azarbarzin², Sara Mariani², \\ Angelo Franciosini ${ }^{3}$, Alessandra Camassa ${ }^{4}$, Thomas Georgeson ${ }^{1}$, \\ Melania Marques ${ }^{2,5}$, Luigi Taranto-Montemurro², Ludovico Messineo², \\ Susan Redline ${ }^{2}$, Andrew Wellman ${ }^{2}$ and Scott A. Sands ${ }^{2}$
}

@ERSpublications

The degree of pharyngeal airflow obstruction varies widely for any given OSA severity (apnoeahypopnoea index) and is challenging to measure. Here we combine information from automated flow shape to accurately estimate the severity of airflow obstruction. http://bit.ly/2uYD0rf

Cite this article as: Mann DL, Terrill PI, Azarbarzin A, et al. Quantifying the magnitude of pharyngeal obstruction during sleep using airflow shape. Eur Respir J 2019; 54: 1802262 [https://doi.org/10.1183/ 13993003.02262-2018].

\section{ABSTRACT}

Rationale and objectives: Non-invasive quantification of the severity of pharyngeal airflow obstruction would enable recognition of obstructive versus central manifestation of sleep apnoea, and identification of symptomatic individuals with severe airflow obstruction despite a low apnoea-hypopnoea index (AHI). Here we provide a novel method that uses simple airflow-versus-time ("shape") features from individual breaths on an overnight sleep study to automatically and non-invasively quantify the severity of airflow obstruction without oesophageal catheterisation.

Methods: 41 individuals with suspected/diagnosed obstructive sleep apnoea (AHI range 0-91 events $\cdot \mathrm{h}^{-1}$ ) underwent overnight polysomnography with gold-standard measures of airflow (oronasal pneumotach: "flow") and ventilatory drive (calibrated intraoesophageal diaphragm electromyogram: "drive"). Obstruction severity was defined as a continuous variable (flow:drive ratio). Multivariable regression used airflow shape features (inspiratory/expiratory timing, flatness, scooping, fluttering) to estimate flow:drive ratio in 136264 breaths (performance based on leave-one-patient-out cross-validation). Analysis was repeated using simultaneous nasal pressure recordings in a subset $(n=17)$.

Results: Gold-standard obstruction severity (flow:drive ratio) varied widely across individuals independently of AHI. A multivariable model (25 features) estimated obstruction severity breath-by-breath $\left(\mathrm{R}^{2}=0.58\right.$ versus gold-standard, $\mathrm{p}<0.00001$; mean absolute error $\left.22 \%\right)$ and the median obstruction severity across individual patients $\left(\mathrm{R}^{2}=0.69, \mathrm{p}<0.00001\right.$; error $\left.10 \%\right)$. Similar performance was achieved using nasal pressure.

Conclusions: The severity of pharyngeal obstruction can be quantified non-invasively using readily available airflow shape information. Our work overcomes a major hurdle necessary for the recognition and phenotyping of patients with obstructive sleep disordered breathing. 


\section{Introduction}

Pharyngeal airflow obstruction, characterised by a mismatch between actual ventilatory flow ("flow") and ventilatory effort ("drive"), is a hallmark of obstructive sleep disordered breathing [1]. For decades, investigators have sought to assess airflow obstruction and consequent flow limitation [2-4] for the purposes of phenotyping obstructive versus central contributions to sleep apnoea $[5,6]$, and for identifying individuals with a low apnoea-hypopnoea index (AHI) who have severe airflow obstruction and symptoms that may warrant treatment $[7,8]$. It is now widely appreciated that the AHI is not a reliable indicator of sleep disordered breathing symptoms or outcomes, with several studies suggesting that the severity of airflow obstruction may be influential [9-15]. A non-invasive means of estimating the severity of pharyngeal obstruction is needed [16].

The field of sleep medicine is now reinvigorating efforts to automatically detect pharyngeal obstruction (i.e. inspiratory flow limitation) based on the flow "shape" of individual breaths [17, 18]. Clinically, pharyngeal obstruction is recognised by expert scientists based on a flattening or scooping (mid-inspiratory dip) of inspiratory flow [19-21], an increase in inspiratory time at the expense of expiratory time [22], and complex flow-limited breath patterns involving intermittent collapse and fluttering [23]. Thus, it is feasible that these features could be combined to estimate the severity of airflow obstruction automatically.

Important progress has already been made: automated methods to detect flow shape features currently form the underlying algorithms within autotitrating continuous positive airway pressure (CPAP) machines [20], yet these algorithms are not publicly available [24]. The main limitation to the available published approaches $[6,18,20,25-28]$ has been the reliance on patient characteristics or subjective expert interpretation to classify presence or absence of obstruction, and most studies have limited methods to a single flow shape feature $[6,20]$.

We have developed and validated a tool that combines flow shape features across multiple known domains (flattening, scooping, timing, fluttering) to estimate airflow obstruction objectively across a continuum of severities. First, we define airflow obstruction based on the ratio of actual ventilation (flow) to the intended ventilation based on calibrated intraoesophageal diaphragm electromyogram (EMG) (drive), i.e. the flow:drive ratio $[29,30]$. Second, flow shape features were calculated in participants with suspected or diagnosed obstructive sleep apnoea $(n=41)$. A host of flow shape features (see the methods section) were used to develop a multivariable model to estimate the severity of pharyngeal airflow obstruction (flow: drive) in individual breaths, and average obstruction severity in individual patients. Finally, we examined the performance of the same multivariable flow shapes model using the nasal pressure signal as a clinically applicable means to quantify obstruction $(\mathrm{n}=17)$.

\section{Methods}

Subjects

43 participants with suspected or diagnosed obstructive sleep apnoea (OSA) attended our sleep research laboratory. All individuals with suspected but not diagnosed OSA reported witnessed snoring/gasping plus daytime sleepiness/fatigue. Patients were free of major comorbidities and were not using medications expected to influence ventilatory control. The study was approved by the Partners internal review board. All participants provided written informed consent before participation. Two participants could not tolerate oesophageal catheterisation (see later), leaving 41 patients with data available for analysis.

\section{Procedure}

In addition to routine polysomnographic signals (electroenchephalography, ECG, thoracoabdominal movements, oximetry), airflow was measured using a pneumotach (Hans Rudolf, Shawnee, KS, USA; Validyne Engineering, Northbridge, CA, USA) attached to an oronasal mask (AirFit small; ResMed Inc., San Diego, CA, USA). To provide gold-standard measurement of ventilatory drive, minimally influenced by changes in airflow [31], each patient was instrumented with an intraoesophageal diaphragmatic EMG catheter (Servo-i Ventilator; Maquet Getinge Group, Wayne, NJ, USA). A subset of patients $(n=17)$

Affiliations: 'School of Information Technology and Electrical Engineering, The University of Queensland, Brisbane, Australia. 'Division of Sleep and Circadian Disorders, Dept of Medicine, Brigham and Women's Hospital and Harvard Medical School, Boston, MA, USA. ${ }^{3}$ Inst. Neur. Timone, Aix-Marseille Université, Marseille, France. ${ }^{4}$ Institut d'Investigacions Biomèdiques August Pi Sunyer (IDIBAPS), Barcelona, Spain. ${ }^{5}$ Laboratorio do Sono, Instituto do Coracao (InCor), Hospital das Clinicas, HCFMUSP, Faculdade de Medicina, Universidade de Sao Paulo, Sao Paulo, Brazil.

Correspondence: Dwayne L. Mann, School of Information Technology and Electrical Engineering, The University of Queensland, GP-South, St Lucia, Brisbane, Queensland, 4072, Australia. E-mail: d.mannduq.edu.au 
underwent simultaneous nasal pressure recording via a modified cannula. Patients slept supine throughout the studies.

The physiological link between airflow obstruction and flow shape

In principle, the flow shape (airflow versus time profile), manifested via pharyngeal obstruction, depends not only on the degree of collapsibility, but also on the underlying ventilatory drive (supplementary figure S1), and thus reflects the ratio of actual airflow (ventilation, referred to as flow) to intended ventilation (ventilatory drive, referred to as drive). Thus, this flow:drive ratio was used as a continuous quantitative measure of the severity of airflow obstruction, scaled so that $100 \%$ indicates a patent airway based on wakefulness $[5,29]$. Note that flow:drive $=50 \%$ indicates obstruction with actual flow equal to just half of the intended flow (drive) consequent to reduced flow and/or increased drive. The flow:drive definition of obstruction is intentionally broad to encompass any form of manifest flow limitation (e.g. Starling resistor "flatness", negative effort dependence "scoopiness"), more complex airway obstruction (e.g. intermittent collapse), and simple linear increases in resistance, all encapsulated by their common effect to reduce ventilation for any given level of ventilatory drive (lowered flow:drive). In principle, flow:drive is equal to the reciprocal of the respiratory system neuromechanical impedance (linear equivalent, resistance and elastance, presented as a fraction of the average wakefulness levels).

Data analysis

Gold-standard measurement of airflow obstruction (flow:drive)

Periods with absent signals, artefact or non-zero CPAP were manually excluded. Breaths scored as obstructive apnoea were automatically excluded from flow shape processing. All other breaths were analysed, regardless of sleep stage (wake, sleep, arousal). Breath-to-breath ventilation was measured using tidal volume $\times$ respiratory rate $\left(\mathrm{L} \cdot \mathrm{min}^{-1}\right)$. Ventilatory drive was calculated using the processed diaphragm EMG excursions, calibrated to L. $\min ^{-1}$ using wakefulness data. Details can be found in the supplementary material. Flow:drive was calculated as ventilation $\left(\mathrm{L} \cdot \mathrm{min}^{-1}\right)$ divided by ventilatory drive $\left(\mathrm{L} \cdot \mathrm{min}^{-1}\right)$ and expressed as a percentage.

\section{Flow shape measures}

To provide a method with broad clinical utility we downsampled airflow data (pneumotach and nasal pressure) to $25 \mathrm{~Hz}$, i.e. American Academy of Sleep Medicine minimum sampling rate [32]. Nasal pressure signals were linearised as appropriate (supplementary figure S2) [30].

We included published features $[6,19,20]$ in addition to novel metrics as candidates. For inclusion, features had to be independent of flow amplitude and able to be calculated without external information (e.g. reference breaths). In total, 85 candidate features were calculated for each breath to reflect aspects of flattening, scooping, asymmetry, breath timing and high-frequency variability (spectral power). Details can be found in the supplementary material (supplementary table S1). Key features are highlighted in the results section.

Features that were not well-characterised using nasal pressure $\left(\mathrm{R}^{2} \leqslant 0.5\right.$ versus pneumotach flow measures, $\sim 40 \%$ of features; supplementary table S2) were excluded, since clinical application (nasal pressure) is a major goal of this work.

Flow-shape estimation of obstruction severity

We adopted a simplified "machine-learning" approach for face-validity and translatability, as follows.

\section{Feature transformation}

To handle nonlinear associations between flow shape features and obstruction severity, we made transformed versions of each feature (square and square-root) and untransformed versions all available for selection.

Multivariable regression to quantify airflow obstruction

Multivariable linear regression with backwards elimination was employed to identify a model (set of feature terms and coefficients) for predicting obstruction severity (flow:drive, continuous variable).

Analysis of nasal pressure

Flow shape features were assessed in 17 patients; the model was used to estimate obstruction severity compared with the gold standard (flow:drive from pneumotach and diaphragm EMG). 


\section{Statistical analysis}

Reported estimates of flow:drive throughout the study were based on a conservative leave-one-patient-out cross-validation procedure, whereby flow:drive estimates from each patient were based on a modified version of the model using only other patients' data. The coefficient of determination $\left(\mathrm{R}^{2}\right)$ was used to assess the strength of the relationship between the gold standard obstruction severity (flow:drive) and the flow shape estimated for each breath (all data wake and sleep). The same approach was used to assess the association between the median obstruction severity (gold standard versus estimated) for each patient (all breaths during sleep only, data in arousals excluded to assess sleep-related obstruction). $\mathrm{R}^{2}>0.5$ was considered a strong association. Mean absolute error was used to describe the expected estimation error. Weighted least-squares was used in regression model development to balance the influence of five severity classes (normal flow:drive >90\%; mild 70-90\%; moderate $50-70 \%$; severe $30-50 \%$, very severe $<30 \%$ ). $\mathrm{p}<0.05$ was considered statistically significant. Multivariable regression assessed whether obstruction severity estimated from flow shape predicted the gold-standard (flow:drive) independent of obstruction frequency (AHI).

\section{Results}

Participant characteristics are summarised in table 1. In total, 136264 breaths in 41 participants were assessed.

\section{Relationship between airflow obstruction severity and OSA severity}

Although we observed an association between median gold-standard obstruction severity (flow:drive) and OSA severity (AHI), the median obstruction severity varied widely for any given obstruction frequency (AHI, residual standard deviation $=23 \%$; figure 1). Some individuals had profound obstruction despite a low AHI.

\section{Multivariable flow shape estimate of airflow obstruction \\ Number of shape features}

Mean absolute error (estimated minus gold standard, cross-validated) decreased with increasing numbers of shape features (beyond 100 features); a model with 25 features represented a sensible compromise between accuracy and complexity (supplementary figures S3 and S4).

\begin{tabular}{|c|c|c|}
\hline & $\begin{array}{l}\text { Comparison to gold } \\
\text { standards }\end{array}$ & $\begin{array}{c}\text { Nasal pressure versus } \\
\text { pneumotach }\end{array}$ \\
\hline Subjects & 41 & 17 \\
\hline \multicolumn{3}{|l|}{ Demographics } \\
\hline Age years & $58 \pm 9$ & $59 \pm 9$ \\
\hline Sex male:female & $25: 16$ & $11: 6$ \\
\hline Race black:white:Asian:other & $12: 28: 0: 1$ & $3: 14: 0: 0$ \\
\hline Body mass index $\mathrm{kg} \cdot \mathrm{m}^{-2}$ & $32.4 \pm 6.6$ & $31.8 \pm 7.5$ \\
\hline Neck circumference cm & $41.3 \pm 4.8$ & $41 \pm 5.2$ \\
\hline Currently treated with CPAP:oral appliance:untreated & $15: 2: 24$ & $2: 1: 14$ \\
\hline \multicolumn{3}{|l|}{ Polysomnography } \\
\hline OSA severity normal:mild:moderate:severe ${ }^{\#}$ & $5: 13: 7: 16$ & $4: 8: 2: 3$ \\
\hline $\mathrm{AHI}$ total events $\cdot \mathrm{h}^{-1}$ & $30.5 \pm 27.4$ & $15.4 \pm 15.7$ \\
\hline$A H I$ non-REM events $\cdot h^{-1}$ & $30 \pm 28$ & $14.5 \pm 16.1$ \\
\hline Central events, non-REM \% respiratory events & $0.8 \pm 3.5$ & $0.5 \pm 1.2$ \\
\hline Hypopnoeas, non-REM \% respiratory events & $64.4 \pm 31.2$ & $80.3 \pm 21.7$ \\
\hline Arousal index, non-REM events $\cdot h^{-1}$ & $50.3 \pm 24.2$ & $42.7 \pm 18.4$ \\
\hline Total sleep time min & $239 \pm 92$ & $249 \pm 80$ \\
\hline Sleep time, spontaneous breathing ${ }^{\Uparrow}$ min & $160 \pm 96$ & $209 \pm 83$ \\
\hline Non-REM $1 \%$ total sleep time & $37 \pm 20$ & $37 \pm 23$ \\
\hline Non-REM $2 \%$ total sleep time & $48 \pm 17$ & $46 \pm 18$ \\
\hline Non-REM $3 \%$ total sleep time & $6 \pm 7$ & $8 \pm 10$ \\
\hline REM \% total sleep time & $9 \pm 8$ & $9 \pm 9$ \\
\hline
\end{tabular}

Data are presented as $n$ or mean \pm SD. CPAP: continuous positive airway pressure; OSA: obstructive sleep apnoea; AHI: apnoea-hypopnoea index; REM: rapid eye movement sleep. \# : normal AHI $<5$ events.h ${ }^{-1}$; mild 5 events $\cdot \mathrm{h}^{-1}<\mathrm{AHI}<15$ events $\cdot \mathrm{h}^{-1}$; moderate 15 events $\cdot \mathrm{h}^{-1}<\mathrm{AHI}<30$ events $\cdot \mathrm{h}^{-1}$; and severe AHI $>30$ events $\cdot \mathrm{h}^{-1}$. Note that the lower $\mathrm{AHI}$ in the nasal pressure subset was not by design; ${ }^{\text {I }}$ sleep time available for analysis without physiological tests (part of previous study). 

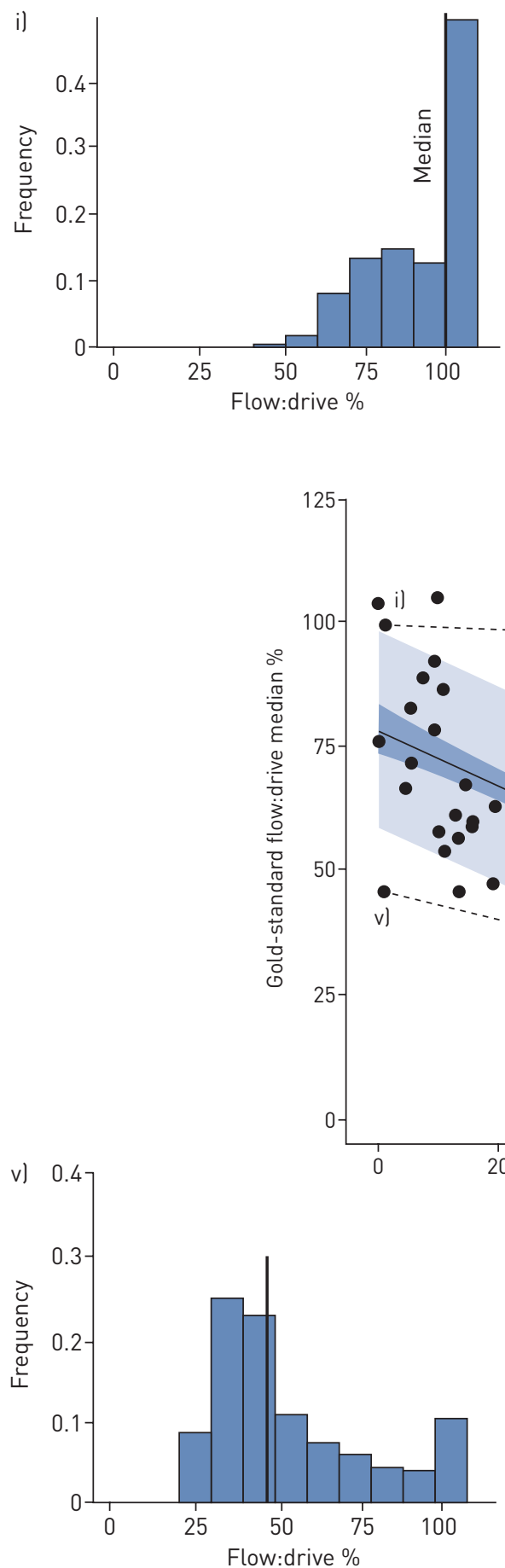
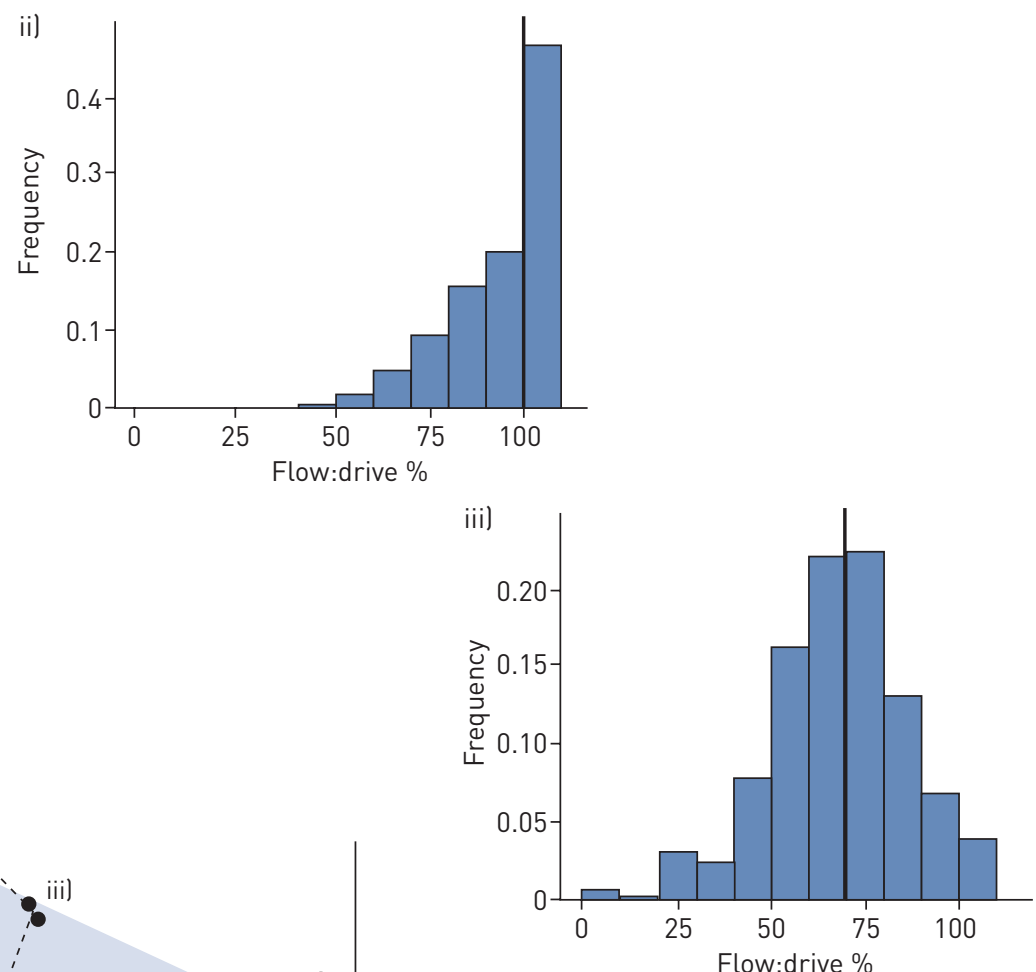

Greater

obstruction

severity

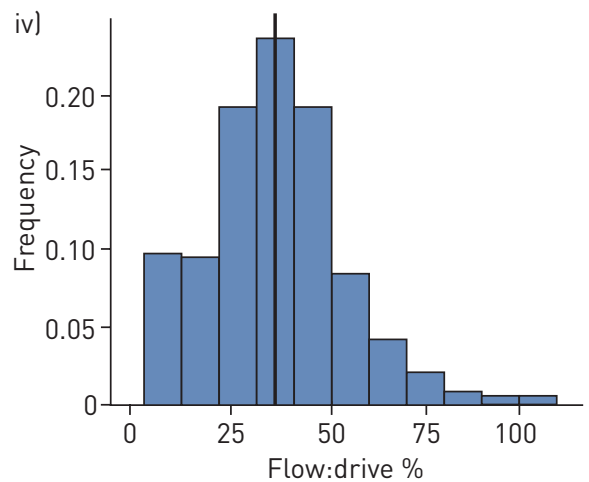

FIGURE 1 Scatter plot (centre) illustrates the association between the severity of airflow obstruction (gold-standard patient median flow:drive ratio) and the frequency of airflow obstruction (apnoea-hypopnoea index (AHI)) during sleep. Only the latter is currently reported clinically. Note that some individuals without sleep apnoea (near-zero AHI) exhibit substantial airflow obstruction (lowered median flow:drive). i-v) (Matched with scatter plot) histograms of airflow obstruction severity (flow:drive) during sleep for selected individuals. i) Normal breathing: this individual has neither sleep apnoea nor airflow obstruction (median flow:drive $=100 \%$ ); ii) moderate sleep apnoea despite normal airflow obstruction (flow:drive=99\%), suggesting a non-obstructive (possibly central) phenotype of sleep apnoea; iii) severe sleep apnoea without severe obstruction (flow:drive $=65 \%$ ), also suggesting a non-obstructive (possibly central) phenotype; iv) severe sleep apnoea with severe airflow obstruction ( $f$ low:drive $=35 \%$ ); v) no sleep apnoea despite a level of airflow obstruction (flow:drive $=46 \%$ ) consistent with severe 0SA, e.g. iv). 


\section{Examples}

Representative traces illustrate concordance between estimated and gold-standard obstruction severity (flow:drive) values in common clinical circumstances (figure 2). Note how knowledge of obstruction provides quantifiable insight into the (otherwise covert) pathophysiology in each example.

\section{Breath-by-breath obstruction}

The estimated obstruction severity for each breath (i.e. continuous flow:drive values predicted from flow shape model; pneumotach signal) was strongly associated with the gold standard flow:drive values (without cross-validation, $\mathrm{R}^{2}=0.63$, mean absolute error $=18 \%$; figure $3 \mathrm{a}$ shows cross-validated results, $\mathrm{R}^{2}=0.58, \mathrm{p}<0.00001$, mean absolute error $\left.=22 \%\right)$.

\section{Patient average obstruction}

The estimated median obstruction severity for each patient during sleep were also strongly associated with gold-standard flow:drive values (without cross-validation, $\mathrm{R}^{2}=0.77$, mean absolute error $=9 \%$; figure $3 \mathrm{~b}$ shows cross-validated results, $\mathrm{R}^{2}=0.69, \mathrm{p}<0.00001$, mean absolute error $\left.=10 \%\right)$. Adjusting for AHI did not diminish the association $(\mathrm{p}<0.00001)$.

\section{Key flow shape measures}

The top five features captured high frequency variability (spectral power) in inspiration and expiration, deviation from a normal rounded contour in inspiration and expiration, and the degree of inspiratory scooping (table 2, figure 4). The complete list of 25 shape features utilised in the final model are described in supplementary table S3.

\section{Nasal pressure analysis}

In total, 62990 breaths with simultaneous nasal pressure (clinical airflow signal) and pneumotach oronasal airflow were assessed. Nasal pressure estimates of flow:drive (same model) were strongly associated with pneumotach derived estimates $\left(\mathrm{R}^{2}=0.80, \mathrm{p}<0.00001\right.$, mean absolute error $=8 \%$; figure $5 \mathrm{a}$, supplementary table S3).

Using nasal pressure, the estimated obstruction severity for each breath $\left(R^{2}=0.48, p<0.00001\right.$, mean absolute error $=23 \%$; figure $5 b)$ and for each patient $\left(R^{2}=0.46, p<0.00001\right.$, mean absolute error $=11 \%$, adjusted for AHI: $\mathrm{p}=0.002$; figure $5 \mathrm{c}$ ) were significantly associated with the gold-standard flow:drive. Note that mean absolute errors were similar to the pneumotach estimates (i.e. $22 \%$ and $10 \%$, respectively).

\section{Discussion}

The current study has developed and validated the first automated method for quantifying the magnitude of pharyngeal airflow obstruction during sleep using airflow shape on a breath-by-breath basis. We provide a simple multifeature model that estimates the breath-by-breath obstruction severity and provides an accurate estimate of average obstruction severity (which we show is considerably different from obstruction frequency, i.e. AHI). Use of nasal pressure was similarly effective, illustrating that the current approach can be readily applied to a routine clinical sleep study. Overall, our study demonstrates that the flow shape can be leveraged to quantify the degree of pharyngeal obstruction during sleep, information that is not otherwise evident when assessing sleep apnoea severity.

\section{Novel physiological insights}

Our study demonstrates that magnitude of pharyngeal airflow obstruction (flow limitation), measured on a continuum (ratio of ventilation to ventilatory drive, flow:drive $[30,33]$ ), influences the clinically observed flow shape in obstructive sleep disordered breathing. First, we showed that theoretically (supplementary figure S1) the airflow shape generated by a flow-limited airway is fundamentally affected by both the properties of the airway (collapsibility, tube law, peak flow) as well as the ventilatory drive or effort, and that the ratio of these aspects (flow:drive) theoretically determines the observed flow shape. Second, by direct measurement in patients we demonstrated that a greater mismatch between flow and drive (airflow obstruction severity) is associated with multiple recognisable flow shape abnormalities (supplementary table S3), including greater signal variability (spectral power) in both inspiration and expiration [23], deviation from a rounded ("sinusoidal") inspiratory flow contour [18], greater scooping [21], increased inspiratory time [6] and greater inspiratory flattening [20]. A brief summary of the highest performing features in the final model are presented in table 2 and illustrated in figure 4 . Third, when combined, flow shape features can explain $\sim 58 \%$ of the variability in flow:drive. Fourth, further analysis revealed that the flow shape associations with flow and drive assessed separately is somewhat weaker $\left(\mathrm{R}^{2}=0.45,0.30\right.$ respectively, compared with 0.58 for flow:drive ratio), demonstrating that it is the mismatch between flow 

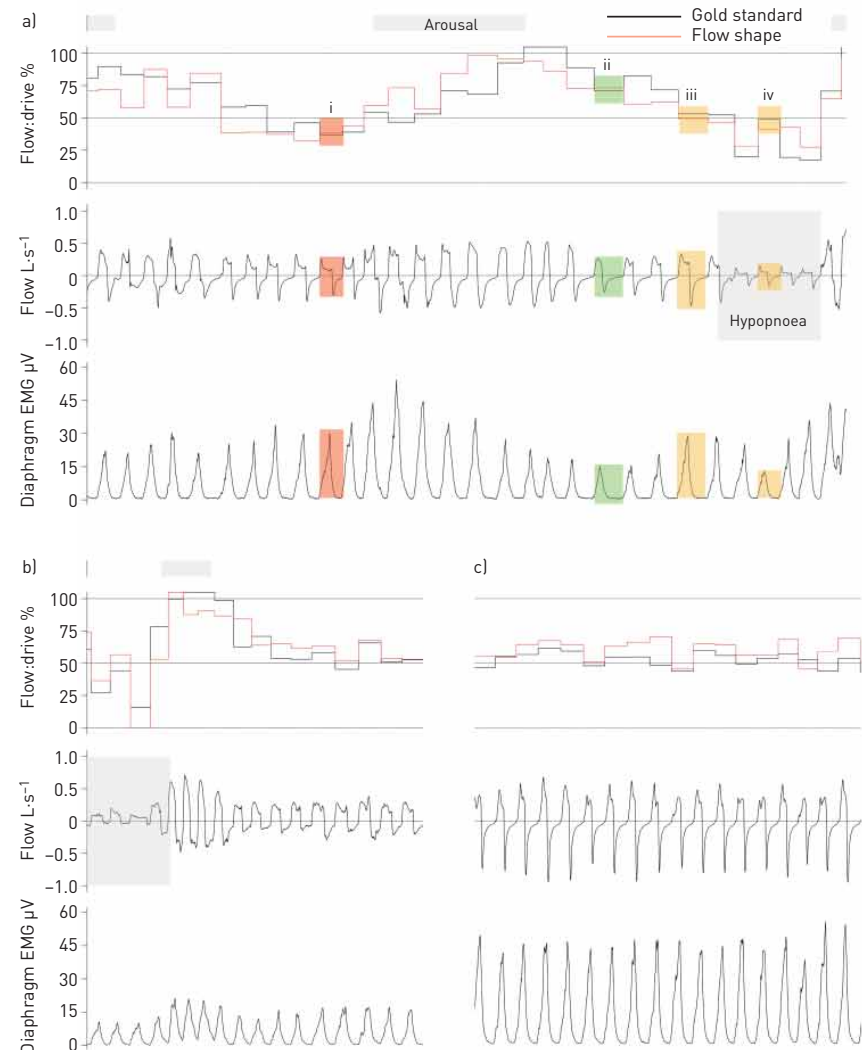

c)

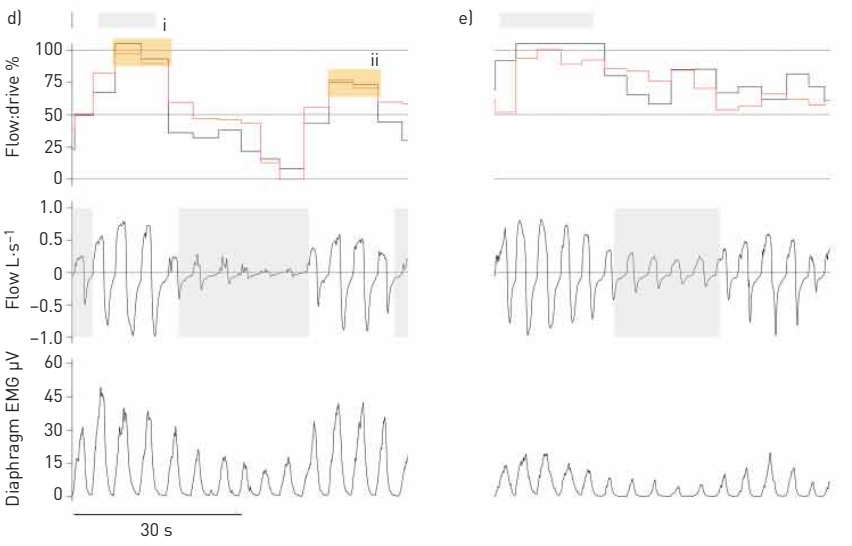

FIGURE 2 Example patterns of airflow obstruction in five individuals (a-e). In each individual, note that the severity of airflow obstruction (flow:drive ratio) estimated based on flow shape matches the gold standard; flow and drive signals are shown separately. a) First, note that in breath (i) the patient does not achieve the airflow that they intended based on drive (lowered flow:drive), which is evident based on the characteristic flow-limited "shape". Compare with breath (ii), where the same flow is achieved but at a lower drive; note the rounded shape when the airway is unobstructed. Second, note that breaths (iii) and (iv) have different magnitudes but identical shapes, consistent with the parallel reduction in flow and drive in (iv), i.e. same obstruction severity. b) Transition from cyclic events to "stable" breathing in a patient with sleep apnoea. Despite so-called stable breathing, pharyngeal obstruction remains substantial lthe patient achieves just half the intended flow, flow:drive $=50 \%$ ), a phenomenon that is recognisable based on flow shape. c) Prolonged obstructed breathing without respiratory events, characterised by increased ventilatory drive, again recognisable based on flow shape. d) At the end of one respiratory event, the airway is fully reopened in association with an arousal (i). A subsequent obstructive event is terminated without complete airway reopening (flow:drive $<100 \%$ ) in the absence of an arousal (ii), illustrating that this patient can achieve marked airflow recovery while maintaining sleep. In this interesting example, we also see the ventilatory drive is falling rather than rising during the event, while the airway is simultaneously becoming obstructed. We note that drive falls towards normal eupnoeic levels, while flow falls to $\sim 10 \%$ of eupnoeic levels, i.e. the reduction in flow is proportionately greater than the reduction in drive, hence obstruction (reduced flow:drive). e) A central hypopnoea, characterised by a reduction in flow that is similar in magnitude to the reduction in drive (diaphragm electromyogram), hence minimal obstruction (and high constant flow:drive). In this case, the airway is more obstructed after (versus during) the event. 

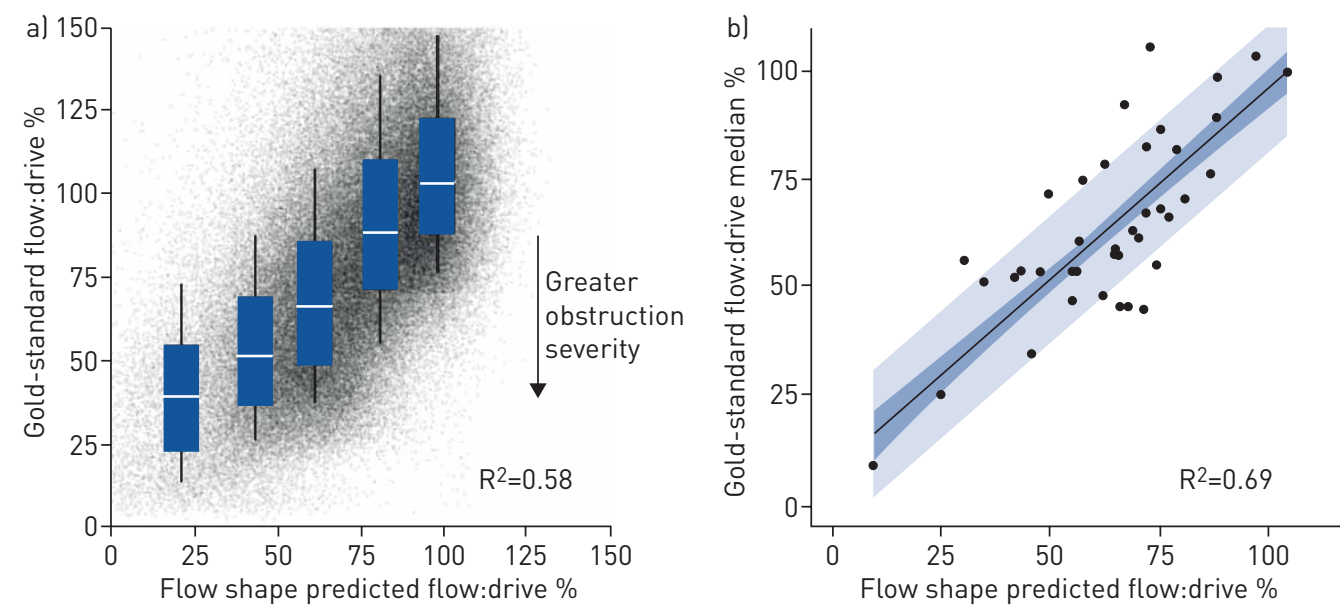

FIGURE 3 Airflow obstruction severity estimated from flow shape (pneumotach) predicts the gold standard in a) individual breaths and b) individual patients. a) Gold-standard versus estimated obstruction severity (flow: drive ratio) for individual breaths (sleep and awake), note the strong association $\left(R^{2}=0.58, p<0.00001\right)$. The box plot overlay shows summary statistics (median, interquartile range; 10th to 90th percentiles) for breaths within each severity classification (normal to very severe). b) Gold-standard versus estimated obstruction severity (flow:drive) for individual patients (median of sleep only data, 73737 breaths), note the strong association $\left(R^{2}=0.69, p<0.00001\right.$, adjusted for apnoea-hypopnoea index $\left.p<0.00001\right)$.

and drive (flow:drive) that best explains the manifest flow shape abnormalities in obstructive sleep disordered breathing.

Our study is the first to demonstrate that combined airflow shape features can be used as a tool to recognise the otherwise concealed severity of airflow obstruction on a breath-by-breath basis. Bivariate analysis showed that there was no single shape feature with high predictive performance across the patient cohort (supplementary table S1). For example, increased inspiratory duty ratio (inspiratory duration $\div$ total breath duration) [28] was modestly associated with obstruction severity $\left(\mathrm{R}^{2}=0.32\right)$, and a well-known

TABLE 2 Selection of highest performing unique features in the multivariable airflow obstruction model

Feature name lin
order of importancel $\begin{gathered}\text { Change with } \\ \text { increasing } \\ \text { obstruction } \\ \text { severity }\end{gathered}$

\begin{tabular}{|c|c|c|c|}
\hline \multirow[t]{2}{*}{ Fluttering } & Power5to12E & Increase & $\begin{array}{l}\text { Power in expiratory flow signal (range } \\
5-12 \mathrm{~Hz} \text { ) }\end{array}$ \\
\hline & Power5to12l & Increase & $\begin{array}{l}\text { Power in inspiratory flow signal (range } \\
5-12 \mathrm{~Hz} \text { ) }\end{array}$ \\
\hline \multirow[t]{3}{*}{ Scooping } & QuadE & Increase & $\begin{array}{l}\text { Area between expiratory flow shape and a } \\
\text { quadratic best fit to } 3 \text { points ( } x, y \text { : } \\
\text { start-expiration, } 0 ; t E / 2, P E F ; \\
\text { end-expiration, } 0 \text { ) }\end{array}$ \\
\hline & QuadI50 & Increase & $\begin{array}{l}\text { Part area between inspiratory flow shape and } \\
\text { a quadratic best fit to } 3 \text { points ( } x, y \text { : start } \\
\text { inspiration, } 0 ; t \mathrm{I} / 2 \text {, PIF; end inspiration, } 0 \text { ). } \\
\text { Area taken is from } 25 \text { th to } 75 \text { th centiles of } \\
\text { inspiratory time }\end{array}$ \\
\hline & AreaUnderPeaksl & Increase & $\begin{array}{l}\text { Area between inspiratory airflow signal and } \\
\text { connected peaks }\end{array}$ \\
\hline
\end{tabular}

Features are in order of importance based on backwards feature elimination (i.e. Power5to12E was the final feature remaining after sequential elimination). The five features listed here are illustrated in figure 4. A simplified five-feature model is provided in the supplementary material (table S5, figures S5 and S6) and performed effectively. tE: expiratory time; PEF: peak expiratory flow; $t \mathrm{l}$ : inspiratory time; PIF: peak inspiratory flow (note PIF was normalised). 

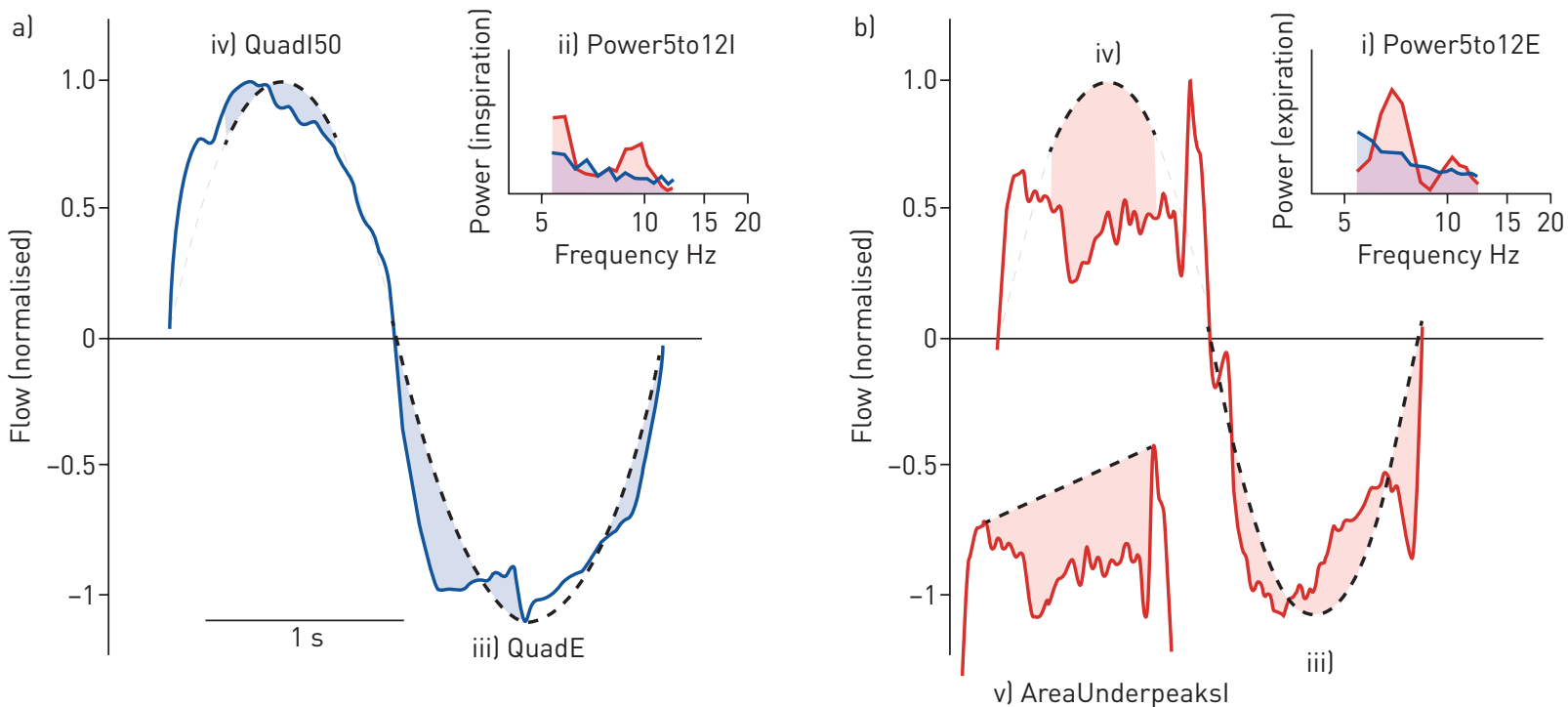

FIGURE 4 The five key features in the multivariable airflow obstruction model. Example a) normal and b) obstructed breaths are shown. Increasing values of these features, labelled $i-v$, indicate greater obstruction. Specifically, (i and ii) Power5to12E and Power5to12I quantify the flow variability (power) in 5-12 Hz range for expiration and inspiration respectively; note the area (power) for the obstructed breath is greater than the area for the normal breath. (iii and iv) QuadE and Quadl50 quantify the discrepancy between the airflow signal and a matched parabola (same peak amplitude, curved dashed lines), for expiration and inspiration, respectively. The absolute difference (areal quantifies the discrepancy (only the middle $50 \%$ of inspiration is used, all data for expiration). (v) AreaUnderPeaksl captures the degree of scooping by quantifying the area under a line joining two inspiratory peaks. A simplified five-feature model is provided in the supplementary material (table S5, figures S5 and S6) and performed effectively.

inspiratory flattening index used for CPAP titration [20] also performed modestly $\left(\mathrm{R}^{2}=0.34\right)$. Combining multiple features was considerably more effective $\left(R^{2}=0.58\right)$, consistent with the knowledge that pharyngeal obstruction manifests as different flow shapes in different patients $[21,23,25]$.

\section{Clinical implications}

A major goal of the field for decades has been a non-invasive clinically applicable automated method to quantify the magnitude of airflow obstruction on individual breaths using a clinically accessible airflow signal (nasal pressure) for the purposes of 1) detecting obstructive sleep disordered breathing (i.e. upper airway resistance syndrome $[12,34,35])$ in patients with minimal overt respiratory events; and 2) facilitating discrimination between obstructive and central phenotypes of sleep apnoea.

It is well recognised that the symptoms of sleep-disordered breathing are not well explained by the frequency of respiratory events (AHI), and that there are a number of symptomatic individuals with severe unrecognised flow limitation (i.e. upper airway resistance syndrome $[12,34,35]$ ) who may benefit from treatment [9-15]. Available evidence has also demonstrated that hallmarks of greater obstruction severity (including increased oesophageal pressure swings, loud snoring) are associated with sleepiness and hypertension independent of the AHI [9-15]. For example, surgical treatment of flow limitation in a small study of children was effective at relieving symptoms [12], CPAP treatment of flow limitation in pregnancy can improve pre-eclampsia [14], and surgical treatment of flow limitation in adults may improve sleepiness [13]. However, the role of obstruction severity in the absence of overt apnoeas and hypopnoeas has remained poorly investigated due to the lack of objective and clinically applicable measurement techniques. Indeed, a recent American Thoracic Society working group [17] emphasised the need to develop an automated open-source algorithm (full model is provided in the supplementary material), with clear recommendations for recording standards (minimum $25 \mathrm{~Hz}$ sampling, DC-coupled, unfiltered), capable of using flow shape to detect airflow obstruction of both non-episodic and episodic nature, that could be related to clinical outcomes in epidemiological studies. Our method addresses and exceeds these requirements by 1) identifying and utilising an objective physiological gold standard (rather than subjective expert-consensus-guided labels); and 2) quantifying the magnitude of pharyngeal obstruction on a continuum.

The differential diagnosis of central versus obstructive sleep apnoea is challenging due to the difficulty of determining the severity of obstruction during sleep. Importantly, treatments for sleep apnoea that target pharyngeal anatomy (CPAP, oral appliances, surgery) are consistently less efficacious in those with a more 
central (or high loop gain) phenotype [36-38]. By definition, this non-obstructive (possibly central) phenotype manifests as the presence of respiratory events despite minimal obstruction severity (e.g. figure 1ii). An estimate such as the median obstruction severity during sleep, which had minimal error ( $10 \%$ pneumotach, $11 \%$ nasal pressure) and was not confounded by AHI, is needed if such patients are to be easily identified for future phenotype-based interventions.

We emphasise that our approach is clinically applicable by design and seeks to obviate the requirement for (invasive) oesophageal catheterisation. Further studies are needed to test the utility of the tool, and determine the role of the severity of airflow obstruction per se in the sequelae of sleep disordered breathing.

\section{Methodological considerations}

There are a number of methodological considerations. Our definition of flow:drive is calibrated based on wake respiratory mechanics, and as such, the calibration value may change across the night. To control for this, the calibration value was extrapolated using a weighted moving-time average to best represent changes across the night. The calibration to wakefulness also means that our gold-standard measure of airflow obstruction is expressed relative to wakefulness (i.e. relative resistance) rather than absolute obstruction (i.e. absolute resistance); thus deficits in wake mechanics will not be detected (e.g. underestimating absolute resistance in obese patients with wake deficits). We emphasise that our goal was to describe sleep-specific changes. Second, we chose a relatively simple feature selection and classification routine (multivariable linear regression with stepwise feature selection) to train and validate our model to ensure face validity and ease of sharing with other investigators. We performed a separate validation study ( $n=29$; details can be found in the supplementary material) that showed that the current method had positive predictive value of $88 \%$ and negative predictive value of $85 \%$ to detect obstructed breaths (in hypopnoeas) versus unobstructed breaths (in arousals). It is possible that further performance improvements could be achieved by the use of more sophisticated classification tools; however, such approaches can obfuscate important discriminatory features. Likewise, combining input from other physiological indicators of airflow obstruction (e.g. respiratory belts, oximetry, snoring sounds, transcutaneous carbon dioxide) may improve model performance [39]. In addition, we note that our method does not require an unusual signal quality: we intentionally used signals downsampled to $25 \mathrm{~Hz}$ to represent a standard clinical polysomnogram (maximising clinical applicability). While increased signal quality might be expected to improve the method, a recent study found that lowering sampling rate to $25 \mathrm{~Hz}$ did not greatly affect ability to detect presence/absence of flow limitation [40], provided that the sampling rate was similar during development and testing.

The current study made no attempt to use flow shape to estimate the site of collapse or to estimate the timing of pharyngeal collapse with respect to the phases of the respiratory cycle. However, we have inherently taken into account, through the various candidate flow shapes that capture aspects of both
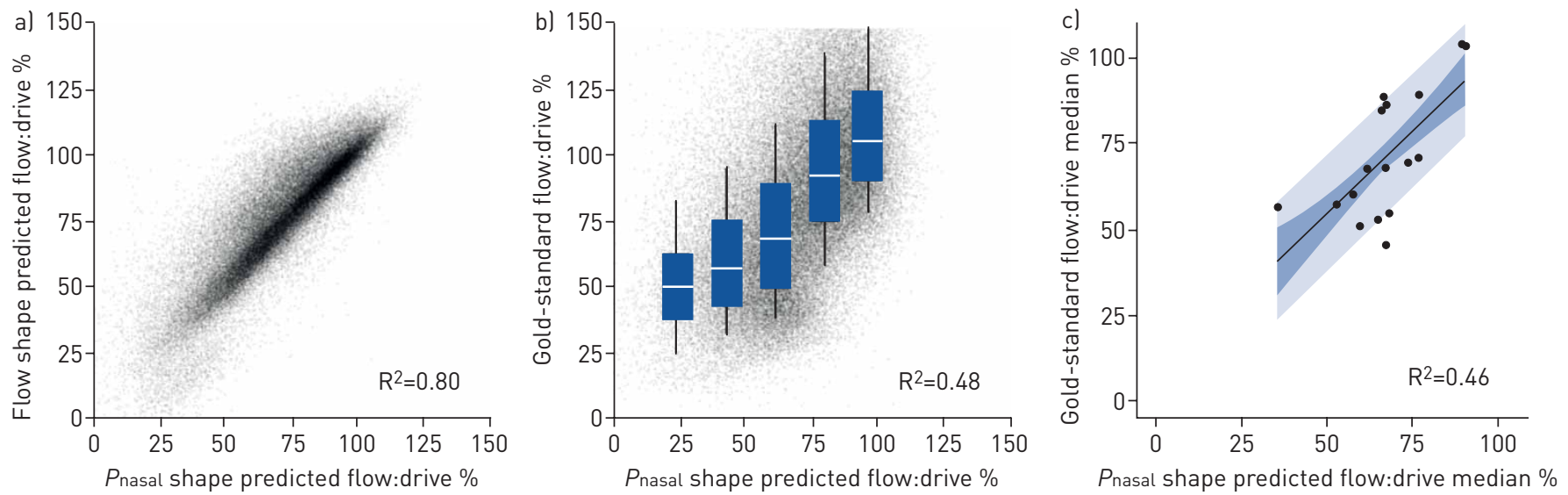

FIGURE 5 Nasal pressure analysis of airflow obstruction. a) Scatter plot showing a very strong association $\left(R^{2}=0.8\right)$ between nasal pressure $(P$ nasal $)$ estimated flow:drive ratio and pneumotach estimated flow:drive values. b) Scatter plot showing a moderate association $\left(R^{2}=0.48\right)$ between nasal pressure estimated flow:drive and gold-standard flow:drive values for individual breaths. The box plot overlay shows summary statistics (median, interquartile range, $10^{\text {th }}$ and $90^{\text {th }}$ percentiles) for breaths within each severity classification. A small reduction in performance (compared to pneumotach flow, figure 3al appears in the distinction between very severe and severe obstruction. c) A moderate association is observed between $P$ nasal estimated median flow:drive and gold-standard patient median flow:drive during sleep, including apnoea breaths $\left(R^{2}=0.46\right.$, $p<0.003$, adjusted for apnoea-hypopnoea index $p<0.003$. Note that in this subset of patients that include simultaneous oronasal pneumotach flow and $P_{\text {nasal }}$ signals, there are very few patients with severe and very severe airflow obstruction as shown by gold-standard flow:drive $<50 \%$. 
inspiratory and expiratory skewness and asymmetry, the understanding that the timing of obstruction is heterogeneous.

Our measure of obstruction severity (flow:drive) does not, by itself capture the balance between central and obstructive contributions to loss of flow during hypopnoeas, or capture the absolute drop in drive per se. Recognition of a central phenotype may require not only specific consideration of flow:drive during events, but also assessment of flow, and the evolution of these measures across the course of events: We would consider hypopnoeas to be of 1) classic "obstructive" manifestation if drive rises while flow falls throughout a hypopnoea, recognisable by a fall in flow:drive that occurs faster in comparison to the fall in flow (i.e. drive increases); and 2) more "central" in manifestation (than classic obstruction) if flow falls in parallel with a fall in drive (with obstruction nonetheless present when flow falls faster than drive, e.g. figure $2 \mathrm{~d}$ ), recognisable by a fall in flow:drive that occurs slower than the fall in flow (i.e. drive decreases). Examples can be found in the supplementary material (supplementary figure S8). Future studies validating the utility of these approaches with respect to clinical outcomes of therapy are warranted.

\section{Conclusions}

We provide a method for the quantification of pharyngeal airflow obstruction that uses information derived entirely from the airflow shape of any given breath. The method compared favourably against a physiological gold standard, on a breath-by-breath basis and when assessing average patient obstruction during sleep. Use of nasal pressure did not yield a substantial deterioration in performance, indicating that our technique is ready for application to routine clinical polysomnography. As such, our work provides tools needed to address the long-standing inability to discriminate between obstructive and non-obstructive manifestations of sleep apnoea, with implications for guiding sleep apnoea therapies. Likewise, it may enable recognition of a disturbing magnitude of pharyngeal airway obstruction in symptomatic non-apnoeic individuals who are presently ineligible for treatment of sleep disordered breathing on the basis of a low AHI.

Author contributions: Study design: D.L. Mann, P.I. Terrill, S.A. Sands; algorithm development: D.L. Mann, A. Azarbarzin, S. Mariani, A. Franciosini, A. Camassa, S.A. Sands; data analysis: D.L. Mann, P.I. Terrill, S.A. Sands; interpretation of results and preparation of the manuscript: all authors.

Conflict of interest: D.L. Mann reports grants from Australian National Health and Medical Research Council (NHMRC) (number 1064163) and the University of Queensland Graduate School (international travel award), during the conduct of the study; personal fees (research scholarship) from The University of Queensland, outside the submitted work. P.I. Terrill reports grants from Australian NHMRC, during the conduct of the study; grants from Hull Family Donation, 2014, outside the submitted work. A. Azarbarzin reports personal fees from Somnifix LLC, outside the submitted work. S. Mariani has nothing to disclose. A. Franciosini has nothing to disclose. A. Camassa has nothing to disclose. T. Georgeson has nothing to disclose. M. Marques has nothing to disclose. L. Taranto-Montemurro reports grants from American Heart Association, personal fees from Novion, Apnimed and Cambridge Sound Management, outside the submitted work. L. Messineo has nothing to disclose. S. Redline reports grants from National Institutes of Health (NIH) grants, grants and personal fees from Jazz Pharma, outside the submitted work. A. Wellman reports grants from NIH/National Heart, Lung, and Blood Institute (NHLBI), grants and personal fees for consultancy from Somnifix, personal fees for consultancy from Cambridge Sound Management, Bayer, Galvani and Nox, grants from Phillips and Sanofi, outside the submitted work; in addition, A. Wellman has a patent 14715829 issued, and has a financial interest in Apnimed Corp., a company developing pharmacological therapies for sleep apnoea. These interests were reviewed and are managed by Brigham and Women's Hospital and Partners HealthCare in accordance with their conflict of interest policies. S.A. Sands reports grants from American Heart Association and NIH, during the conduct of the study; personal fees from Cambridge Sound Management, Nox Medical, Merck and Apnimed, outside the submitted work.

Support statement: This work was supported by a grant from the National Health and Medical Research Council of Australia (NHMRC; 1064163) and the National Institutes of Health (NIH; R01HL128658). D.L. Mann was supported by a University of Queensland Research Scholarship and Graduate School International Travel Award. S.A. Sands was supported by the American Heart Association (15SDG25890059), an NHMRC Early Career Fellowship and R.G. Menzies award (1053201), an American Thoracic Society foundation unrestricted grant. S. Mariani was supported as a coinvestigator of NIH grants (R24HL114473, U01HL119991, and R35HL135818). Funding information for this article has been deposited with the Crossref Funder Registry.

\section{References}

Ayappa I, Rapoport DM. The upper airway in sleep: physiology of the pharynx. Sleep Med Rev 2003; 7: 9-33.

2 Calero G, Farre R, Ballester E, et al. Physiological consequences of prolonged periods of flow limitation in patients with sleep apnea hypopnea syndrome. Respir Med 2006; 100: 813-817.

3 de Godoy LB, Palombini LO, Martinho Haddad FL, et al. New insights on the pathophysiology of inspiratory flow limitation during sleep. Lung 2015; 193: 387-392.

4 Palombini LO, Tufik S, Rapoport DM, et al. Inspiratory flow limitation in a normal population of adults in São Paulo, Brazil. Sleep 2013; 36: 1663-1668. 
5 Randerath WJ, Treml M, Priegnitz C, et al. Evaluation of a noninvasive algorithm for differentiation of obstructive and central hypopneas. Sleep 2013; 36: 363-368.

6 Mooney AM, Abounasr KK, Rapoport DM, et al. Relative prolongation of inspiratory time predicts high versus low resistance categorization of hypopneas. J Clin Sleep Med 2012; 8: 177-185.

7 Guilleminault C, Stoohs R. Arousal, increased respiratory efforts, blood pressure and obstructive sleep apnoea. J Sleep Res 1995; 4: 117-124.

8 Chandra S, Sica AL, Wang J, et al. Respiratory effort-related arousals contribute to sympathetic modulation of heart rate variability. Sleep Breath 2013; 17: 1193-1200.

9 Nakano H, Hirayama K, Sadamitsu Y, et al. Mean tracheal sound energy during sleep is related to daytime blood pressure. Sleep 2013; 36: 1361-1367.

10 Stoohs R, Guilleminault C. Snoring during NREM sleep: respiratory timing, esophageal pressure and EEG arousal. Respir Physiol 1991; 85: 151-167.

11 Guilleminault C, Stoohs R, Shiomi T, et al. Upper airway resistance syndrome, nocturnal blood pressure monitoring, and borderline hypertension. Chest 1996; 109: 901-908.

12 Guilleminault C, Winkle R, Korobkin R, et al. Children and nocturnal snoring: evaluation of the effects of sleep related respiratory resistive load and daytime functioning. Eur J Pediatr 1982; 139: 165-171.

13 Newman JP, Clerk AA, Moore M, et al. Recognition and surgical management of the upper airway resistance syndrome. Laryngoscope 1996; 106: 1089-1093.

14 Edwards N, Blyton DM, Kirjavainen T, et al. Nasal continuous positive airway pressure reduces sleep-induced blood pressure increments in preeclampsia. Am J Respir Crit Care Med 2000; 162: 252-257.

15 Pépin JL, Guillot M, Tamisier R, et al. The upper airway resistance syndrome. Respiration 2012; 83: 559-566.

16 Rapoport DM. On beyond zebra (and the apnea-hypopnea index) in obstructive sleep apnea. Am J Respir Crit Care Med 2018; 197: 1104-1106.

17 Pamidi S, Redline S, Rapoport D, et al. An Official American Thoracic Society Workshop report: noninvasive identification of inspiratory flow limitation in sleep studies. Ann Am Thorac Soc 2017; 14: 1076-1085.

18 Zhi YX, Vena D, Popovic MR, et al. Detecting inspiratory flow limitation with temporal features of nasal airflow. Sleep Med 2018; 48: 70-78.

19 Hosselet JJ, Norman RG, Ayappa I, et al. Detection of flow limitation with a nasal cannula/pressure transducer system. Am J Respir Crit Care Med 1998; 157: 1461-1467.

20 Teschler $\mathrm{H}$, Berthon-Jones $\mathrm{M}$, Thompson $\mathrm{AB}$, et al. Automated continuous positive airway pressure titration for obstructive sleep apnea syndrome. Am J Respir Crit Care Med 1996; 154: 734-740.

21 Genta PR, Sands SA, Butler JP, et al. Airflow shape is associated with the pharyngeal structure causing OSA. Chest 2017; 152: 537-546.

22 Onal E, Lopata M. Respiratory timing during NREM sleep in patients with occlusive sleep apnea. J Appl Physiol 1986; 61: 1444-1448.

23 Azarbarzin A, Marques M, Sands SA, et al. Predicting epiglottic collapse in patients with obstructive sleep apnoea. Eur Respir J 2017; 50: 1700345.

24 Brown LK. Autotitrating CPAP: how shall we judge safety and efficacy of a "black box"? Chest 2006; 130: 312-314.

25 Aittokallio T, Malminen JS, Pahikkala T, et al. Inspiratory flow shape clustering: an automated method to monitor upper airway performance during sleep. Comput Methods Programs Biomed 2007; 85: 8-18.

26 Clark SA, Wilson CR, Satoh M, et al. Assessment of inspiratory flow limitation invasively and noninvasively during sleep. Am J Respir Crit Care Med 1998; 158: 713-722.

27 Ayappa I, Norman RG, Krieger AC, et al. Non-invasive detection of respiratory effort-related arousals (REras) by a nasal cannula/pressure transducer system. Sleep 2000; 23: 763-771.

28 Schneider H, Krishnan V, Pichard LE, et al. Inspiratory duty cycle responses to flow limitation predict nocturnal hypoventilation. Eur Respir J 2009; 33: 1068-1076.

29 Catcheside $\mathrm{P}$, Reynolds K, Stadler D, et al. Ventilatory effort versus output in obstructive sleep apnoea assessed via the respiratory system equation of motion. J Sleep Res 2014; 2014: 59.

30 Sands SA, Edwards BA, Terrill PI, et al. Phenotyping pharyngeal pathophysiology using polysomnography in patients with obstructive sleep apnea. Am J Respir Crit Care Med 2018; 197: 1187-1197.

31 Luo YM, Wu HD, Tang J, et al. Neural respiratory drive during apnoeic events in obstructive sleep apnoea. Eur Respir J 2008; 31: 650-657.

32 Iber C, Ancoli-Israel S, Chesson ALJ, et al. The AASM Manual for the Scoring of Sleep and Associated Events: Rules, Terminology and Technical Specifications. 1st Edn. Westchester, IL, American Academy of Sleep Medicine, 2007.

33 Catcheside PG, Reynolds K, Stadler D, et al. Ventilatory effort versus output in obstructive sleep apnea assessed by the respiratory system equation of motion. Sleep Biol Rhythms 2014; 12: Suppl. 1, 59.

34 Downey R 3rd, Perkin RM, MacQuarrie J. Upper airway resistance syndrome: sick, symptomatic but underrecognized. Sleep 1993; 16: 620-623.

35 Douglas NJ. Upper airway resistance syndrome is not a distinct syndrome. Am J Respir Crit Care Med 2000; 161: 1413-1415.

36 Bradley TD, Logan AG, Kimoff RJ, et al. Continuous positive airway pressure for central sleep apnea and heart failure. N Engl J Med 2005; 353: 2025-2033.

37 Edwards BA, Andara C, Landry S, et al. Upper-airway collapsibility and loop gain predict the response to oral appliance therapy in patients with obstructive sleep apnea. Am J Respir Crit Care Med 2016; 194: 1413-1422.

38 Joosten SA, Leong P, Landry SA, et al. Loop gain predicts the response to upper airway surgery in patients with obstructive sleep apnea. Sleep 2017; 40: zsx094.

39 Kaplan V, Zhang JN, Russi EW, et al. Detection of inspiratory flow limitation during sleep by computer assisted respiratory inductive plethysmography. Eur Respir J 2000; 15: 570-578.

40 Camassa A, Franciosini, A, Sands, SA, et al. Validating an algorithm for automatic scoring of Inspiratory Flow Limitation within a range of recording settings. In: 40th Annual International Conference of the IEEE Engineering in Medicine \& Biology Society (EMBC), 2018; Honololu, HI, USA, 2018. 